\title{
3D-hydrodynamics of colliding winds in massive binaries
}

\author{
Rolf Walder \\ Steward Observatory, University of Arizona, \\ 933 North Cherry Avenue, Tucson, AZ 85721, USA
}

\author{
Doris Folini \\ Observatoire de Strasbourg, F-67000 Strasbourg, la France
}

\begin{abstract}
The pinwheel nebulae observed in some WC-binaries essentially mirror a transport phenomenon. We show the importance of the central wind collision zone in setting the 'initial conditions' for this transport. In order to understand some of the newly observed features, we postulate that standard theory of colliding flows must be extended by considering radiative breaking, heat-conduction, and the clumped character of the winds. We suggest that clumped winds can be modeled by highly compressible turbulence and outline some consequences for the physics of the wind collision zone. With regard to dust production we argue that the system center is the only location where dust nucleation can happen.
\end{abstract}

\section{Introduction}

In recent years, fantastic new observations have brought new insights into phenomena related to colliding winds in massive star binaries. IR pinwheel nebulae (Tuthill, Monnier \& Danchi 1999; Marchenko et al. 2002, Monnier, Tuthill \& Danchi 2002b) give insights into the circum-stellar environment on a scale ranging up to 1000 stellar separation lengths. New observations in wavelengths from $\mathrm{X}$ to IR have revealed that some points of the classical theory of binary windwind collision must be revised. To give only two examples: in contrast to classical theory, the shocks of the wind-wind interaction in WR 140 at periastron passage are highly radiative (Marchenko \& Moffat, private communication). Monnier et $a l$. (2002a) report that the non-thermal emission region in WR-binaries is generally larger than classical theory predicts. This seems to match with the X-ray emission of V444 Cyg measured by Corcoran et al. (1996), who see gas with, compared to predictions, a larger spatial extension but a lower temperature.

In this paper we comment on these developments from a theoretical point of view. In Section 2, we discuss how the observed pinwheels are connected with colliding winds and why they form Archimedian spirals. Section 3 is devoted to a glimpse at physical processes beyond classical theory. In particular, we consider the collision of two highly compressible flows as a model for the collision of clumped hot star winds. Some arguments why such a model favors the production of dust are given. We summarize in Section 4. 

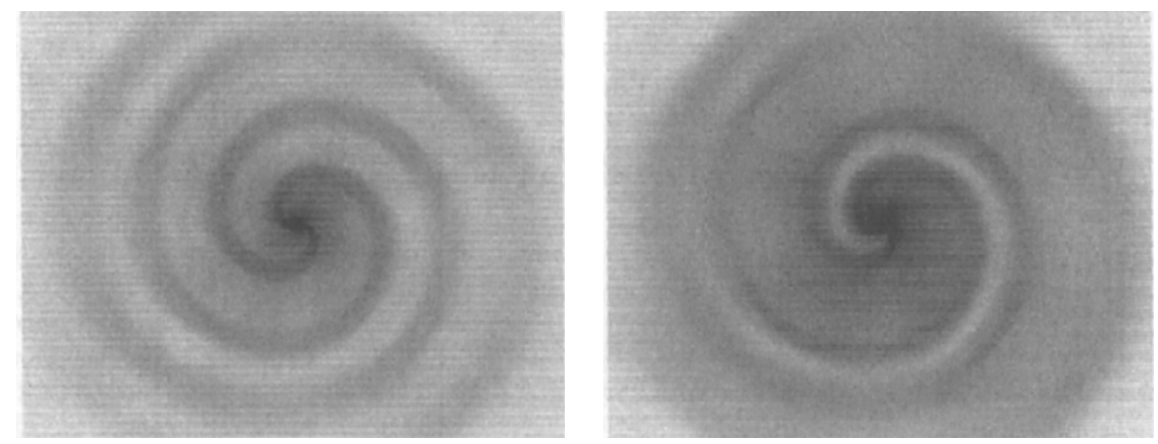

Figure 1. Comparison of spiral pattern of models A (left) and B (right) in the orbital plane on a scale of $333 \mathrm{AU}$, roughly 200 times the stellar separation. Density is shown as a logarithmic grey-scale plot between $\log \rho=3$ (white) and $\rho=7.5$ and higher (black).

\section{Shocks and large scale spiral patterns}

\subsection{Circular orbits}

In some WC binaries, pinwheel nebulae are observed in IR dust emission (see Tuthill et al. these Proceedings). The pinwheels are shaped like an Archimedian spiral and interpreted as tracers of the wind-wind interaction zone. We will discuss the geometry and properties of the observed spirals with the help of two toy-models. In both models, the orbital period of the binary system is 90 days and the mass of both stars is $23 \mathrm{M}_{\odot}$. Both stars have winds with a terminal velocity of $1500 \mathrm{~km} \mathrm{~s}^{-1}$. The stars in model A have mass-losses of $1 \cdot 10^{-6}$ and $1.8 \cdot 10^{-6} \mathrm{M}_{\odot} \mathrm{yr}^{-1}$, those in model $\mathrm{B} 1 \cdot 10^{-6}$ and $1 \cdot 10^{-5} \mathrm{M}_{\odot} \mathrm{yr}^{-1}$. The simulations are purely hydrodynamical and radiative cooling is neglected. Figure 1 shows the resulting spirally shaped structures of the two models. All simulations in this paper were performed with our A-MAZA code (Walder \& Folini 2000).

But let us start with a look at the very central region of the system. As illustrated in Figure 2, the interaction of the two winds actually takes place only in the region between the two stars, perhaps on a scale 2-3 times the separation of the two stars. Only there the stellar winds get shocked, raising the temperature to a very high value, and compressing the matter strongly. In this central region, the interaction of the winds results in a tilted shock cone, the opening angle of which is given by the ratio of the two wind-momenta, its tilt by the ratio of the wind and orbital velocities. Within the interaction zone, the shocked material is re-accelerated to approximately the wind-speed. The main difference between models A and B, the much narrower shock cone of model B, is due to the different mass-losses. The physics of this central region is well investigated and we have something like a 'standard theory', mostly worked out by Usov (1991, 1992), Stevens, Blondin \& Pollock (1992), and Walder (1995).

On scales larger than approximately two times the stellar separation no further hydrodynamical action takes place, neither between the two winds, nor between the winds and the material streaming outwards off the central interac- 

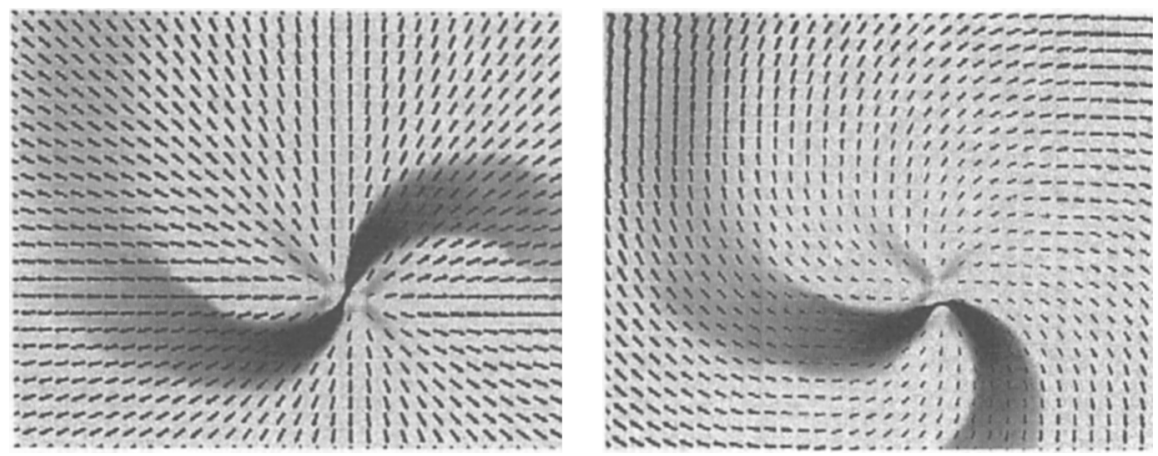

Figure 2. Velocity field in the orbital plane on a scale of $60 \mathrm{AU}$ of model A in the observers frame (left) and of model B in the co-rotating frame (right). Temperature is shown as a logarithmic grey-scale between $\log T=4.6$ (white) and 7.6 (black). The jet-like structures in the center are numerical artifacts.

tion zone. In the coordinate system co-rotating with the two stars, the velocity field gets completely tangential to the spirals emerging from the central region (Figure 2, right). In the observers frame the gas moves radially outward (Figure 2, left). The spiral is a wave pattern arising from the superposition of the gas and orbital velocity. The shape of the spirals corresponds to the tangential curve of the co-rotating velocity field: $v_{r}=v_{\text {wind }}=$ const., $V_{\theta}(r)=v_{\text {corot }}=\omega r$, where $P=2 \pi \omega$ is the period and $r$ the distance to the center of mass of the binary system. Both spiral arms in the orbital plane are Archimedian spirals. However, since the central interaction shock-cone of model B has a much smaller opening angle, the two arms in this model lie much closer together. Model B resembles much more a WR-system. If the dust in the spiral shell is optically thick, we see, looking at the system head-on, only one spiral which completely confines the O-star wind. If the dust shell is not completely optically thick two spiral arms can in principle be seen due to line of sight effects. If the line of sight is arbitrary, a correct interpretation of the observations needs complicated radiative transfer.

We summarize that the wind-wind interaction takes only place within 2-3 stellar separations. From there the material is transported outwards without any further interaction, the transport path describing Archimedian spirals. Along this transport, the gas may cool, recombine, dust grains may grow, but there are no more external effects on the flow apart from stellar radiation. The 'initial conditions' of this flow are set in the very center of the system.

\subsection{Eccentric orbits at the example of WR 140}

We proceed to eccentric systems and present preliminary results of a simulation of WR $140(e=0.82)$, a system which at each periastron passage produces dust. We use wind- and system-parameters as in the axisymmetric simulations of Stevens, Blondin \& Pollock (1992), which were derived by Williams et al. (1990). Smooth winds are considered. Optically thin radiative cooling is included but affects the flow only on large scales as the cooling time is considerably longer than dynamical time-scales in the center of the system. 

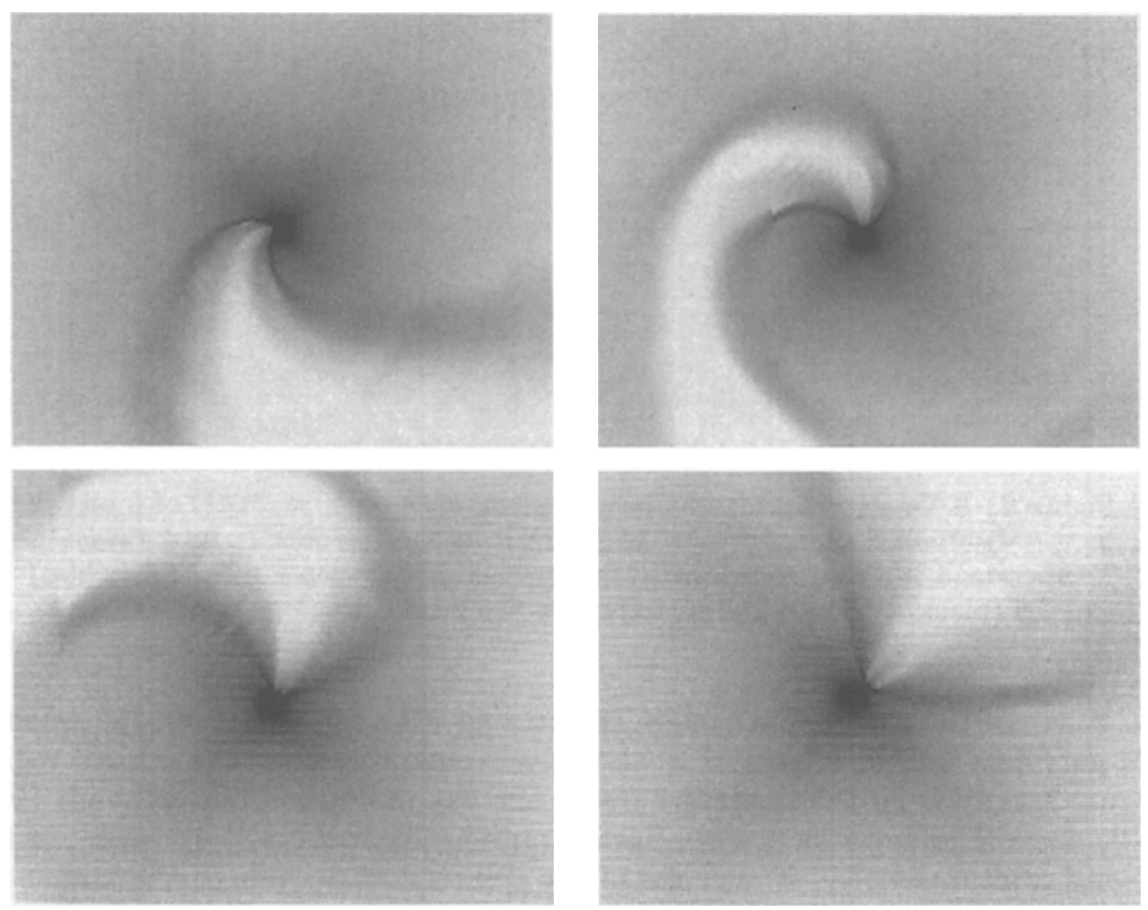

Figure 3. Density in logarithmic grey-scale (white: 5.35 , black: 10.35) in the orbital plane on a scale of $333 \mathrm{AU}$ of a simulation of WR 140 at phases $\phi=0.00$ (periastron, upper left), 0.02 (upper right), 0.039 (lower left), and 0.08 (lower right). The stars move clockwise. A video-animation of this simulation can be copied at http://ww. astro.phys.ethz.ch/staff/walder/private. The data are available from the authors upon request.

Our simulation shows that also in this highly eccentric system the shocks and the associated high compression rate are located only in the center of the system. There are no secondary shocks due to the orbital motion. However, the shape and location of the spiral arms are highly variable. The spiral reacts with the corresponding time-delay to the change in orbital velocity of the stars. Its asymmetric shape is a general feature of eccentric systems, causing interesting effects such as the asymmetric X-ray light curve of $\gamma^{2} \mathrm{Vel}$ and occultation effects at quadrature (Walder, Folini \& Motamen 1999; Folini \& Walder 2002).

\section{Some elements beyond classical wind-wind-collision theory}

As said in the introduction, 'classical' theory cannot explain some of the new observations. In our opinion, at least three major new elements must be incorporated into the theory, which, however, are not as 'handy' as the old analytical formulae. The first element is radiative breaking (Gayley, Owocki \& Cramner 1997), the second is heat conduction (Myasnikov \& Zhekov 1998; Motamen, Walder \& Folini 1999), and the third is the clumpy character of hot star winds on which we concentrate here. For a further discussion of the importance and, 


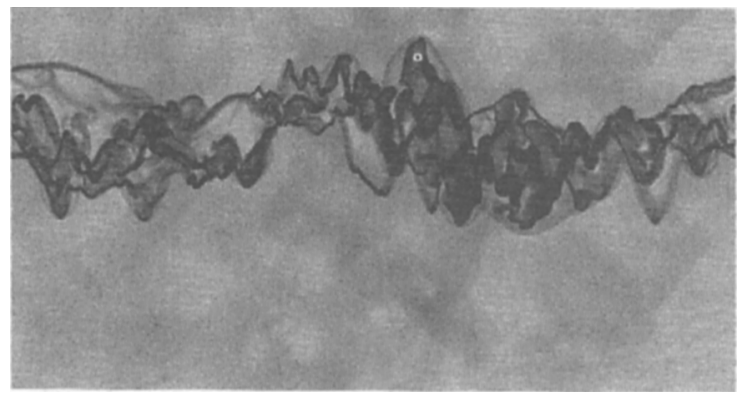

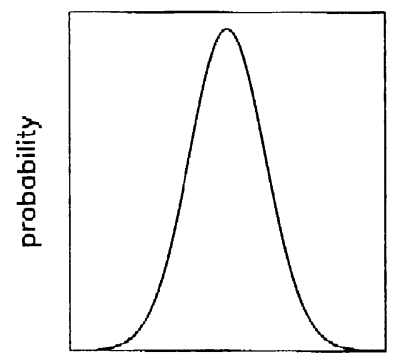

$\log$ [part.dens.]

Figure 4. Colliding supersonically turbulent winds (from top and bottom) and their interaction zone in planar geometry, shown in density as a logarithmic grey-scale (white: 1, black: 4) (left panel). The typical time-averaged donsity distribution in highly compressible turbulence is log-normal (right panel). Mean value and FWHM depend on the flow parameters.

in particular, of the complex interplay of these physical processes we refer to our recent review (Folini \& Walder 2000).

\subsection{Collision of clumped winds}

The first question which must be clarified is the nature of the clumped hot star winds. Two different pictures are under discussion. The canon ball picture in which the clumps are disconnected from the flow and move ballistically through it. And then the supersonic turbulence picture in which the clumps are dynamically confined, transient structures (high and low density) with typical life times on the order of their sound crossing time.

While we do not completely rule out that single clumps exist, we favor the picture of highly compressible turbulence for the generic case. Our argumentation starts with two strong, however astonishing facts: the steady-state analysis of moving atmospheres and winds of hot stars in terms of complex co-moving frame radiative transfer calculations produces synthetic spectra which fit extraordinary well with observed spectra. However, an ad-hoc clumping factor must be introduced to achieve these marvelous results. On the other hand, we know from the analyses by Owocki, Castor \& Ribicki (1988) and Owocki (1999) that line-driven winds are highly unstable and time-dependent, producing variable structures in the wind consisting of high-density knots and voids.

These two, on the first glance, contradictory facts can be brought together within the frame of highly compressible (or supersonic) turbulence. It has been shown by different authors (Mac Low et al. 1999, Vázquez-Semadeni, Gazol \& Scalo 2000) that the time-averaged density (over several crossing times) in such a flow has a log-normal distribution (Figure 4, right), ranging typically over several orders of magnitude in density. The mean density is typically reduced by a factor of 3-10 as compared to a smooth flow. So far, these theoretically predicted wind-structures could hardly be observationally resolved. (We are not speaking of more permanent wind-features like DACs, which most certainly have a different origin, likely linked to processes in the stellar interior.) 
Although such a picture seems plausible, we do not yet know the exact characteristics of such turbulence in stellar winds. This must be the subject of future research which includes the radiation field and the radial acceleration. However, we find it useful to present in Figure 4 the interaction of two purely hydrodynamical supersonically turbulent flows in planar symmetry. As for radiative colliding smooth flows, the interaction zone is supersonically turbulent itself and resembles interaction zones as observed in PNe (Grosdidier, Acker \& Moffat 2001) and as simulated in binaries (Stevens, Blondin \& Pollock 1992). As shown by Walder \& Folini (2002), the cooling is significantly enhanced when clumped flows collide. Moreover, it seems possible that big clumps $\left(z R_{\odot}\right)$ can survive within the hot shocked environment without being evaporated by the $\mathrm{X}$-ray photons or energetic thermal electrons.

\subsection{Notes on the production of dust}

We argue that the region with the by far best conditions to produce first dust nuclei is the very central one. As discussed in much more detail in Walder \& Folini (2002), only there we can hope to find high enough densities, large enough optical depths to shield the energetic stellar photons, and fast enough cooling times to obtain conditions necessary for dust nucleation as discussed by Cherchneff et al. (2000). However, a quantitative analysis has still to be done.

For WR 140 Walder \& Folini (2002) have shown that winds with reasonable clumping indeed lead to radiative shocks at periastron passage. The observations of Moffat and Marchenko 2002 (private communication) now show clear evidence for radiative shocks between phase 0.99 and 0.02 . Consequently, at these phases, densities of up to more than $10^{13} \mathrm{~cm}^{-3}$ can be reached at the stagnation point of the colliding flows. Typical transport velocities out of the center are on the order of the wind-velocities; Williams et al. (1990) estimate $2860 \mathrm{~km} \mathrm{~s}^{-1}$ in the plane of the sky. Therefore, at phase 0.02 , dust will fill the spiral shell from the center out to nearly $100 \mathrm{AU}$. Once cooling becomes insufficient again, the production of dust stops but the already present dust rushes further outwards. At phase $\phi=0.04$ (see Figure 3) at which Monnier, Tuthill \& Danchi (2002b) have taken their first snapshot, the dust shell is no longer connected over the tip of the spiral but lies on a segment of its mantle, approximately 150-200 AU away from the center.

\section{Summary and conclusions}

Spirally shaped wave patterns are tracers of the wind-wind interaction which takes place within 2-3 stellar separation only. Their generic shape is purely determined by the ratio of the wind velocity and stellar orbital velocity. In eccentric systems, the shape of the spirals is variable and reflects - at each location in space - time-delayed the variable orbital velocity of the stars.

For a correct physical description of the central interaction zone radiative breaking, heat conduction, and the clumpy character of hot star winds must be considered. We have considered the collision of the supersonically turbulent flows as a model for the collision of clumped hot star winds and have shown that the interaction of such flows results in a turbulent interaction zone similar to the interaction zone of colliding smooth flows. Cooling, however, is enhanced. We 
concluded that the most favorable location for dust nucleation is in the central region of the system, the only location the wind-wind interaction takes place.

Acknowledgments. Support for this work was provided by the Scientific Discovery through Advanced Computing (SciDAC) program of the US-DOE, grant number DE-FC02-01ER41184. R.W. thanks the Institut für Astronomie at ETH Zürich for its hospitality to provide a part-time office. D.F. was supported by a grant of the Swiss National Science Foundation, grant number 8220-056553.

\section{References}

Cherchneff, I., Le Teuff, Y.H., Williams, P.M., Tielens, A.G.G.M. 2000, A\&A 357, 572

Corcoran, M.F., Stevens, I.R., Pollock, A.M.T., Swank, J.H., Shore, S.N., Rawley, G.L. 1996, ApJ 464, 434

Folini, D., Walder, R. 2002, in: A.F.J. Moffat \& N. St-Louis (eds.), Interacting Winds from Massive Stars, ASP-CS 260, 605

Folini, D., Walder, R. 2000, in: H. Lamers \& A. Sapar (eds.), Thermal and Ionization Aspects of Flows from Hot Stars: Observations and Theory, ASP-CS 204, 267

Gayley, K.G., Owocki, S.P., Cranmer, S.R. 1997, ApJ 475, 786

Grosdidier, Y., Acker, A., Moffat, A.F.J. 2001, A\&A 370, 51

Mac Low, M.-M., Klessen, R., Burkert, A., Smith, M. 1999, in J. Franco \& A. Carraminana (eds.), Interstellar Turbulence (Cambridge: CUP), p. 256

Marchenko, S.V., Moffat, A.F.J., Vacca, W.D., Coté, S., Doyon, R. 2002, ApJ (Letters) 565 , L59

Monnier, J.D., Greenhill, L.J., Tuthill, P.G., Danchi, W.C. 2002a, ApJ 566, 399

Monnier, J.D., Tuthill, P.G., Danchi, W.C. 2002b, ApJ (Letters) 567, L137

Motamen, S.M., Walder, R., Folini, D. 1999, in: K.A. van der Hucht, G. Koenigsberger \& P.R.J. Eenens (eds.), Wolf-Rayet Phenomena in Massive Stars and Starburst Galaxies, Proc. IAU Symp. No. 193 (San Franciso: ASP), p. 378

Myasnikov, A., Zkekov, S. 1998, MNRAS 300, 686

Owocki, S.P. 1999, in: B. Wolf, O. Stahl \& A.W. Fullerton (eds.) Variable and Nonspherical Stellar Winds in Luminous Hot Stars, Proc. IAU Coll. No. 169 (Berlin: Springer), Lecture Notes in Physics 523, 294

Owocki, S.P., Castor, J.I., Ribyicki, G.B. 1988, ApJ 335, 914

Stevens, I.R., Blondin, J.M, Pollock, A.M.T. 1992, ApJ 386, 265

Tuthill, P.G., Monnier, J.D., Danchi, W.C. 1999, Nature 398, 487

Tuthill, P.G., Monnier, J.D., Danchi, W.C., Turner, N.H. 2003, these Proceedings

Usov, V.V. 1992, ApJ 389, 635

Usov, V.V. 1991, MNRAS 252, 49

Vázquez-Semadeni, E., Gazol, A., Scalo, J. 2000, ApJ 540, 271

Walder, R., Folini, D. 2002, in: A.F.J. Moffat \& N. St-Louis (eds.), Interacting Winds from Massive Stars, ASP-CS 260, 595

Walder, R., Folini, D. 2000, in: H. Lamers \& A. Sapar (eds.), Thermal and Ionization Aspects of Flows from Hot Stars: Observations and Theory, ASP-CS 204, 281

Walder, R., Folini, D., Motamen, S.M. 1999, in: K.A. van der Hucht, G. Koenigsberger \& P.R.J. Eenens, (eds.), Wolf-Rayet Phenomena in Massive Stars and Starburst Galaxies, Proc. IAU Symp. No. 193 (San Franciso: ASP), p. 298

Walder, R. 1995, in K.A. van der Hucht \& P.M. William (eds.), Wolf-Rayet Stars: Binaries, Colliding Winds, Evolution, Proc. IAU Symp. No.163, (Dordrecht: Kluwer), p. 420 
Williams, P.M., van der Hucht, K.A., Pollock, A.M.T., Florkowski, D.R., van der Woerd, H., Wamsteker, W.M. 1990, MNRAS 243, 662

\section{Discussion}

CherepashchuK: New information about slow acceleration of WR winds appeared recently. Did you take into account this fact in your 3D modeling?

WALDER: Not in the models I have presented today. So far, there are no models unifying all physical processes.

DWARKADAS: You talked about large-scale, supersonic turbulence. However, most simulations such as those by Owocki, show small-scale, microturbulence, but not large-scale structures. Do you think it is necessary to really have large-scale structures, and if so how are they formed? Would such structure be visible through observations?

WALDER: This is probably a misunderstanding. My view is that - in the vicinity of the star - the size of the turbulent structures are smaller than the size of the star. The clumps I have applied in my model have a diameter comparable to the sun.

MOFFAT: In the image you showed of M1-67, one cannot necessarily say that the WR wind is turbulent on its basis. The structures we see in M1-67 are likely all the result of instabilities in the process of the fast WR wind colliding with the previous slow LBV/RSG wind, whether the winds are clumped or not.

WALDER: I agree with this interpretation. It is, however, a nice illustration of my view of clumped winds. Both winds and interaction shells are highly compressibly turbulent, although the characteristics of the turbulence are certainly different in the two cases. Also the ISM looks very similar in density contrast and cell-like structures.

FELDMEIER: Clumped winds of OB or WR stars are often described by a filling factor which may be constant or some function of radius. Is there a way to describe compressible turbulence, where small and large blobs are present simultaneously in the Kolmogorov spectrum, using an effective filling factor?

WALDER: In planar, even weakly magnetized material $\left(\beta_{\text {plasma }}=a_{\text {sound }} / a_{\text {alfven }} \gtrsim 1\right)$, it was shown that the density distribution is log-normal (in a temporal mean), i.e., the density-probability-function is a Gaussian on a logarithmic density axis. The distribution of the size of the structures is harder to quantify, but certainly you observe large differences in the size of the structures. To my knowledge, there is no quantitative investigation of highly compressible turbulence in radially outflowing material. But different theoretical arguments may lead to the proposition that the structures increase in size with increasing radius as long as the turbulence can effectively be driven. A note on the spectrum: normally the spectrum is splitted into a compressible part ( $\operatorname{rot} \vec{v}=0$ ) and a solenoidal part (div $\vec{v}=0)$. The solenoidal part is indeed Kolmogorov in stationary turbulence, the compressible part, however, follows $\epsilon \propto k^{-2}$.

SonNEBORN: The mixing of $\mathrm{H}$ in the cooling clumps may result in $\mathrm{H}_{2}$ formation in addition to dust. This may be seen in $2.1 \mu$ IR emission (shocked $\mathrm{H}_{2}$ ), or in the far-UV Lyman and Werner bands. Far-UV absorption in particular is a very sensitive probe of the pressure of $\mathrm{H}_{2}$ in the line of sight of the star. This would be a good diagnostic of $\mathrm{H}$ mixing in the wind-wind interface. 
WALDER: Thanks. The models would allow for mixing to some degree, but we cannot yet make quantitative predictions. The dust people argue that without mixed-in hydrogen the production of dust is not favored.

PitTARD: You mentioned the importance of including thermal conduction into the hydrodynamic models of colliding winds. Is it possible that the high temperature shock precursor will destroy those clumps, perhaps through conductive evaporation?

WALDER: If you adopt the picture of only one clump and if this clump is neither very dense nor very large, then (but only then!) it will probably evaporate due to thermal conduction. However, if you consider highly compressible turbulence, the picture changes. Even after the wind material has entered the precursor, it moves still supersonically, although with a lower Mach number. I would therefore expect the turbulence to continue, especially as it can be forced by the undisturbed wind. But certainly, the diffusive process damps the turbulence to a certain degrce. We certainly need quantitative models of this process.

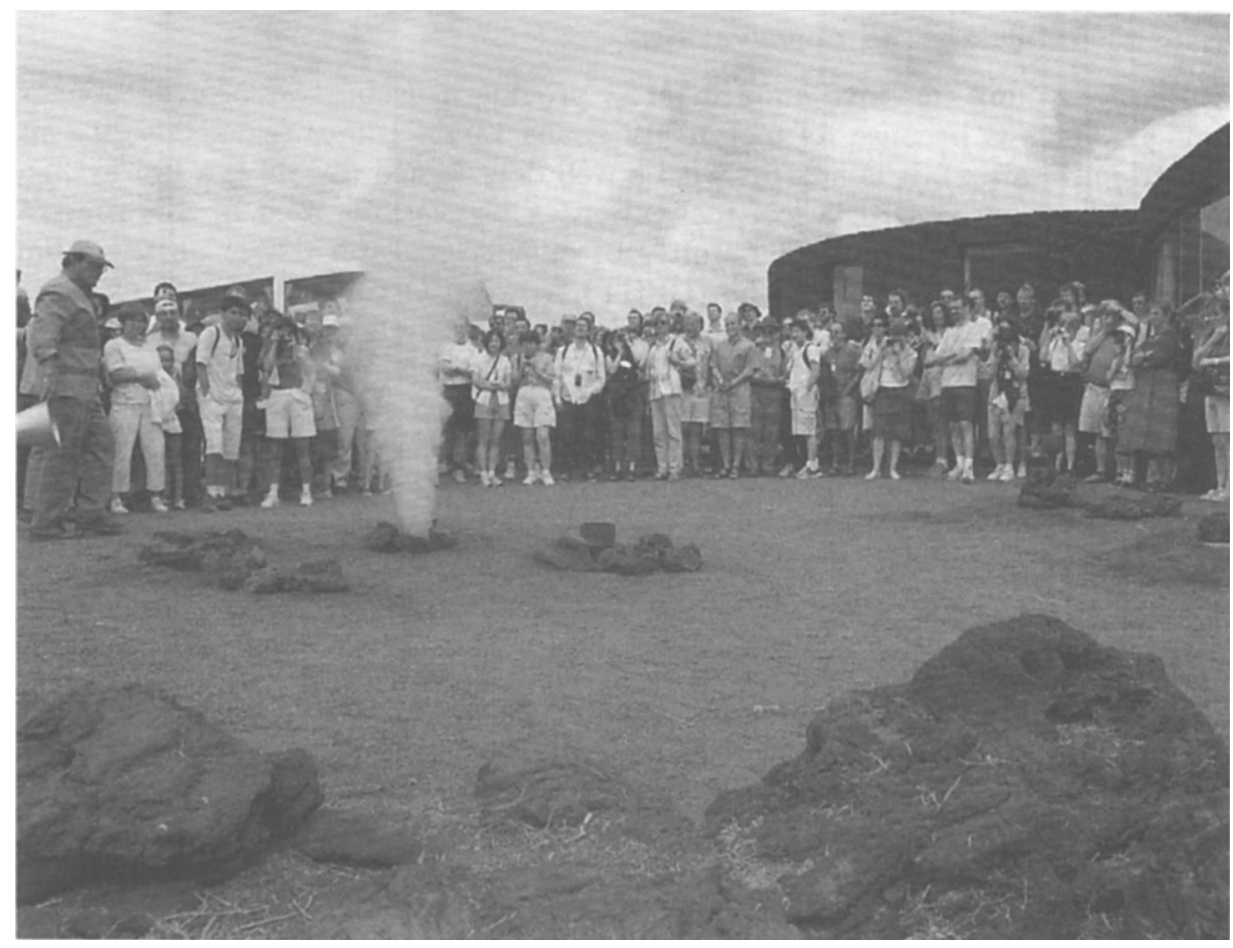

3D-hydrodynamics at work in Timanfaya National Park 